\title{
Public awareness of tetralogy of Fallot after Jimmy Kimmel Live! television episode: A cross-sectional analysis
}

\author{
Benjamin Greiner ${ }^{1}$ (D), Abraham Lee ${ }^{1}$, Jake Checketts $^{2}$ and Micah Hartwell ${ }^{3}$ \\ ${ }^{1}$ University of Texas Medical Branch, Department of Internal Medicine, Galveston, Texas, ${ }^{2}$ Oklahoma State University Medical \\ Center, Department of Orthopaedic Surgery, Tulsa, Oklahoma, and ${ }^{3}$ Oklahoma State University Center for Health Sciences, \\ Department of Psychiatry and Behavioral Sciences, Tulsa, Oklahoma \\ ${ }^{\star}$ Corresponding author: Email: ben.greiner10@gmail.com, Phone: (409) 772-2653.
}

(Received 21 May 2020; Revised 13 July 2020; Accepted 15 July 2020)

\begin{abstract}
Background: Persons with rare disorders, such as tetralogy of Fallot, often feel socially isolated due to poor public awareness of the disorder. On 1 May 2017, Jimmy Kimmel aired a segment on Jimmy Kimmel Live! highlighting the impact of tetralogy of Fallot on his son and how the public can learn more about the disorder.

Methods: We tracked public interest in tetralogy of Fallot using Google Trends and Twitter after the episode and constructed an autoregressive integrated moving average algorithm to calculate search volumes had Kimmel not aired the episode.

Results: Google searches and the number of Tweets for tetralogy of Fallot increased by $3063.27 \%$ and $4672.62 \%$, respectively, above expected.

Conclusions: Our findings indicate that television talk shows may represent strong outlets for increasing public awareness of rare disorders.
\end{abstract}

Key words: Public awareness; tetralogy of Fallot; congenital heart disease

\section{Introduction}

Rare diseases affect nearly 30 million Americans and present a significant physical, financial, and emotional impact on patients which is further compounded by lack of research and public awareness (Griggs et al., 2009). Unfortunately, the psychological effect of having a rare disease often plays an equally large role in co-occurring feelings of despair, lack of therapeutic hope, social isolation, and discrimination (von der Lippe et al., 2017). Thus, increasing public awareness may have a significant impact on the overall health of persons with rare diseases.

One study analyzed the effects of season releases of a popular streaming show including a main actor having cleidocranial dysplasia, a disorder affecting roughly 1 per million people, on public awareness and found a 94\% increase in Google searches following the episode's release (Johnson et al., 2020). This suggests actors may play a role in educating the public on rare diseases. On 1 May 2017, Jimmy Kimmel Live! aired an episode in which Kimmel explained that his son, William Kimmel, was born with tetralogy of Fallot - a rare disorder of the heart classically defined as the combination of pulmonary stenosis, ventricular septal defect, overriding aorta, and right ventricular hypertrophy. Our study objective was to analyze the increases in search volume and Tweets after the episode was released to investigate the effects of the entertainment industry on public awareness of tetralogy of Fallot.

\footnotetext{
(c) The Author(s), 2020. Published by Cambridge University Press. This is an Open Access article, distributed under the terms of the Creative Commons Attribution licence (http://creativecommons.org/licenses/by/4.0/), which permits unrestricted re-use, distribution, and reproduction in any medium, provided the original work is properly cited.
} 
Google Trend of Tetralogy of Fallot and ARIMA Forecasts

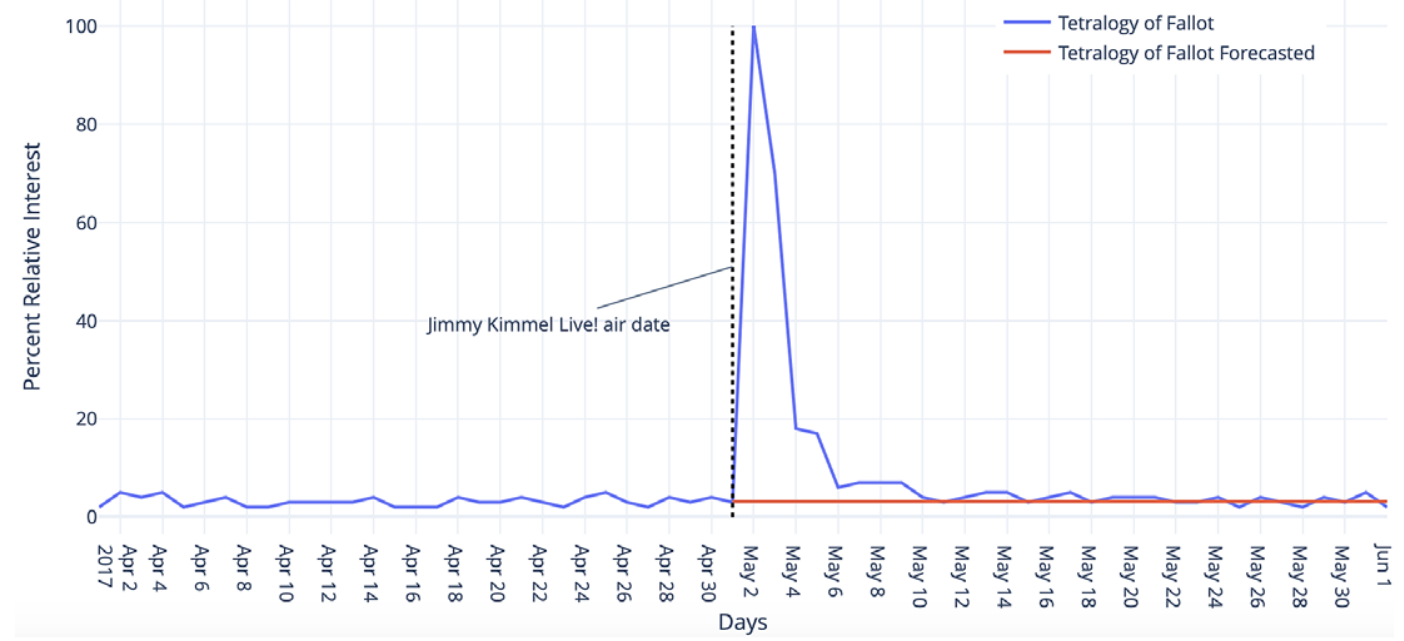

Volume of Daily Tweets for Tetralogy of Fallot with ARIMA Forecasts

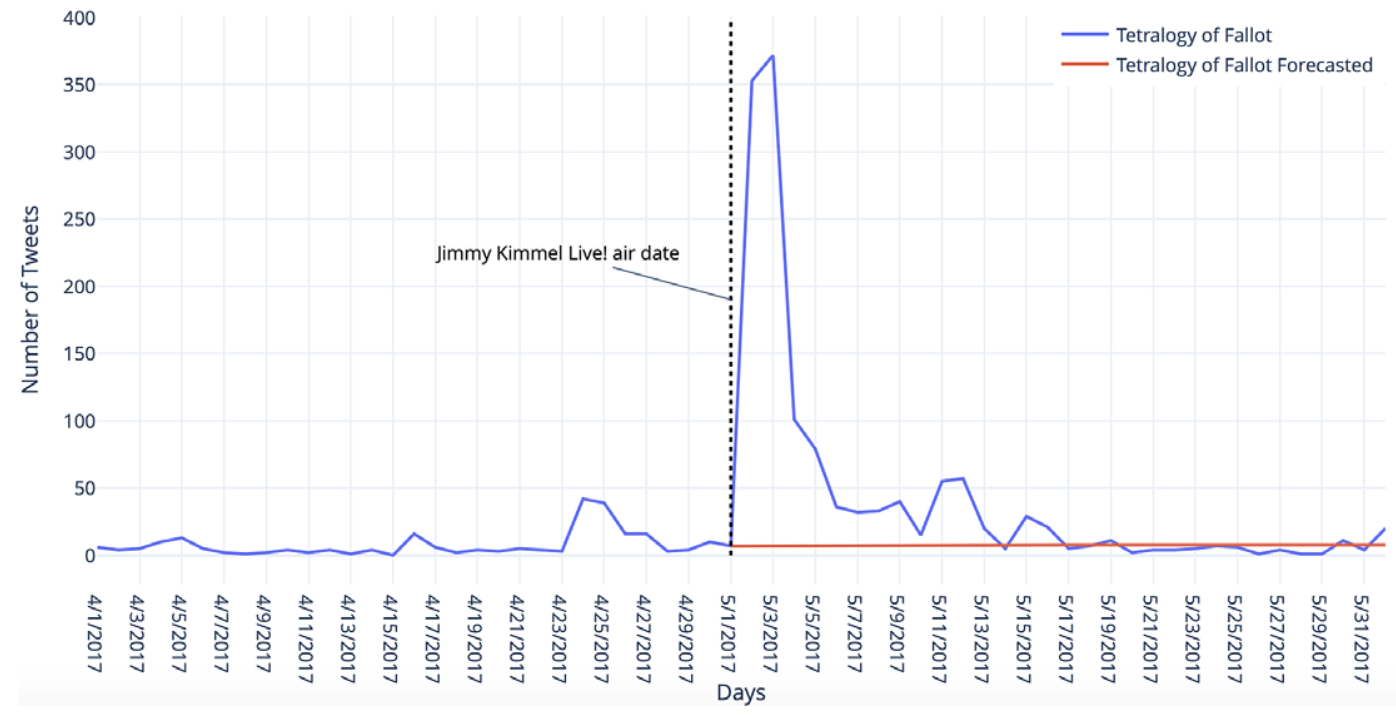

Figure 1. Figure 1 shows the relative search interest, number of Tweets, and the forecasted trends for "Tetralogy of Fallot" by month. Jimmy Kimmel Live air date is indicated by the vertical dashed line.

\section{Methods}

We performed a cross-sectional study of Google Trends (GoogleTrends.com) and Twitter using Sprout Social (SproutSocial.com) - the two most frequently used platforms for health and information, from March 1 through June 1, 2017, for "tetralogy of Fallot." The time frame was selected to limit confounding variables. Google Trends utilizes relative search volume which is scaled from $0-100$ with 100 representing the highest peak volume within the timeframe. To analyze expected search and Tweet volume had Kimmel not publicly discussed tetralogy of Fallot, we constructed forecasting models (Hyndman \& Khandakar, 2008) using autoregressive integrated moving average (ARIMA) algorithms in R (version 3.6.1) with parameters $\mathrm{p}, \mathrm{d}$, and $\mathrm{q}$ determined by the auto.arima function in the Forecast package using 
Akaike information criterion (AICc) adjusted for small samples. For the Google Trends data, these parameters were set as 0,0 , and 0 which inherently predicts the historical mean. These parameters were set at 0,0 , and 1 for the Twitter data.

\section{Results}

Following the episode of Jimmy Kimmel Live!, there were significant increases in Google searches and Tweets. The day after the episode aired (May 2, 2017), relative search interest from Google Trends for "Tetralogy of Fallot" peaked 96.84 (95\% CI 94.94 - 98.74) points above the forecasted value (3.16; 95\% CI 1.26 - 5.06); an increase of $3063.27 \%$ (Figure 1). The number of tweets mentioning "Tetralogy of Fallot" peaked on May 3, 2017 with a total of 364.21 (95\% CI 345.03 - 383.38) tweets above the forecasted value (7.79; 95\% CI -11.38 - 26.97); an increase of $4672.62 \%$.

\section{Discussion}

This study found a significant increase in public awareness of tetralogy of Fallot following an episode of Jimmy Kimmel Live! in which the host discusses his son's rare birth condition. Studies have found increases in public awareness following actors' heightened media attention and their respective conditions (Johnson et al., 2020). However, this study is the first, to our knowledge, to analyze the effect of talk shows on increasing public awareness of health conditions. Furthermore, one of the authors (JC) has been diagnosed with tetralogy of Fallot and notes an anecdotal increase in awareness, support, and curiosity of this condition from lay people who frequently cite the airing of Jimmy Kimmel announcing his son's diagnosis. In light of these findings, as well as current evidence, it can be suggested that increasing awareness of rare diseases may improve peer support, reduce symptom to diagnosis time, increase research funding, and expand treatment options (von der Lippe et al., 2017).

Our study is not without limitations. First, our current study is unable to gauge retention of awareness of tetralogy of Fallot. Second, although Google and Twitter are the most widely used platforms for lay person health information retrieval (Antheunis et al., 2013), they may not accurately represent public interest in tetralogy of Fallot which may have skewed our results. Additionally, though our study demonstrated increased interest, we were unable to determine if this interest was translated into true knowledge and understanding of tetralogy of Fallot. However, it is the author's feeling that these findings may represent a novel method to decrease stigma and increase public awareness of tetralogy of Fallot and other rare diseases. Therefore, we recommend physicians and those with the appropriate platforms consider the use or support of talk shows (or other media) to aid in the dissemination of knowledge regarding rare diseases.

Data suppository statement. The data that support the findings of this study are openly available from GoogleTrends.com and SproutSocial.com.

Financial support. This research received no specific grant from any funding agency, commercial or not-for-profit sectors.

Conflicts of interest. None.

Author contributions. BG and $\mathrm{MH}$ carried out the experiment. BG wrote the manuscript with support from AL, JC, and $\mathrm{MH}$. BG, AL, JC, and MH analyzed the data. BG and MH performed statistical analyses. $\mathrm{MH}$ helped supervise the project. BG and $\mathrm{MH}$ conceived the original idea.

Role of Funding Source. This study was not funded.

Conflict of Interest. We declare no conflicts of interest.

Supplementary Materials. To view supplementary material for this article, please visit http://dx.doi.org/10.1017/exp.2020.34. 


\section{References}

Antheunis, M. L., Tates, K., \& Nieboer, T. E. (2013). Patients' and health professionals' use of social media in health care: Motives, barriers and expectations. Patient Education and Counseling, 92, 426-431. https://doi.org/10.1016/j pec.2013.06.020.

Griggs, R. C., Batshaw, M., Dunkle, M., Gopal-Srivastava, R., Kaye, E., Krischer, J., Nguyen, T., Paulus, K., Merkel, P. A., \& Rare Diseases Clinical Research Network. (2009). Clinical research for rare disease: Opportunities, challenges, and solutions. Molecular Genetics and Metabolism, 96, 20-26. https://doi.org/10.1016/j.ymgme.2008.10.003

Hyndman, R. J., \& Khandakar, Y. (2008). Automatic time series forecasting: Forecasting functions for time series and linear models. Journal of Statistical Software, 27, 8-12. https://doi.org/10.18637/jss.v027.i03.

Johnson, A. L., Torgerson, T., Cooper, C., Khojasteh, J., \& Vassar, M. (2020). Public awareness of cleidocranial dysplasia after season releases of stranger things. JAMA Otolaryngology-Head \& Neck Surgery, 146, 377-378. https://doi.org/10.1001/ jamaoto.2019.4791.

von der Lippe, C., Diesen, P. S., \& Feragen, K. B. (2017). Living with a rare disorder: A systematic review of the qualitative literature. Molecular Genetics and Genomic Medicine, 5, 758-773. https://doi.org/10.1002/mgg3.315.

Cite this article: Greiner B, Lee A, Checketts J, Hartwell M (2020). Public awareness of tetralogy of Fallot after Jimmy Kimmel Live! television episode: A cross-sectional analysis Experimental Results, 1, e26, 1-7. https://doi.org/10.1017/exp.2020.34 


\section{Peer Reviews}

Reviewing editor: Dr. Marc Henrion ${ }^{1,2}$

${ }^{1}$ Malawi-Liverpool-Wellcome Trust Clinical Research Programme, Statistical Support Unit, Queen Elizabeth Central Hospital, PO Box 30096, Blantyre, Malawi

${ }^{2}$ Liverpool School of Tropical Medicine, Clinical Sciences, Pembroke Place, Liverpool, United Kingdom of Great Britain and Northern Ireland, L3 5QA

This article has been accepted because it is deemed to be scientifically sound, has the correct controls, has appropriate methodology and is statistically valid, and met required revisions.

doi:10.1017/exp.2020.34.pr1

Review 1: Public awareness of tetralogy of Fallot after Jimmy Kimmel Live! television episode: A cross-sectional analysis

Reviewer: Dr. Michael Nevels

University of St Andrews, Biomolecular Sciences Building, Fife, United Kingdom of Great Britain and Northern Ireland, KY16 9ST

Date of review: 30 June 2020

(C) The Author(s), 2020. Published by Cambridge University Press This is an Open Access article, distributed under the terms of the Creative Commons Attribution licence (http://creativecommons.org/licenses/by/4.0/), which permits unrestricted re-use, distribution, and reproduction in any medium, provided the original work is properly cited.

Conflict of interest statement. Reviewer declares none

Comments to the Author: This is a somewhat minimal but original study. The question and conclusion are clear, the methods appear to be sound (although I cannot comment on the statistical methods) and the limitations have been discussed.

1. More than just two sources (Google Trends and Twitter) should be considered for the study.

2. Change "with" to "within" in "[...] highest peak volume with timeframe"?

3. Split "Studies have found [...]" into two sentences: "Studies have found [...] conditions. However, this study [...]".

4. Reference 3 is incomplete.

5. Revise spelling of "TheforecastPackage forR" in reference 5.6. A figure legend has to be provided

7. Figure panels should be labeled "A" and "B".

\section{Score Card}

Presentation

Is the article written in clear and proper English? (30\%)

Is the data presented in the most useful manner? (40\%)

Does the paper cite relevant and related articles appropriately? (30\%) 
Does the abstract correctly embody the content of the article? (25\%)

Does the introduction give appropriate context? (25\%)

Is the objective of the experiment clearly defined? (25\%)

Analysis

Does the discussion adequately interpret the results presented? (40\%)

Is the conclusion consistent with the results and discussion? (40\%)

Are the limitations of the experiment as well as the contributions of the experiment clearly outlined? (20\%) 
Review 2: Public awareness of tetralogy of Fallot after Jimmy Kimmel Live! television episode: A cross-sectional analysis

Reviewer: Dr. Mark Wass

University of Kent, Biosciences, Canterbury, United Kingdom of Great Britain and Northern Ireland, CT2 7NZ

Date of review: 12 July 2020

(C) The Author(s), 2020. Published by Cambridge University Press This is an Open Access article, distributed under the terms of the Creative Commons Attribution licence (http://creativecommons.org/licenses/by/4.0/), which permits unrestricted re-use, distribution, and reproduction in any medium, provided the original work is properly cited.

Conflict of interest statement. Reviewer declares none

Comments to the Author: The manuscript reports analysis of google searches and Tweets about Tetralogy of Fallout, following it being discussed by Jimmy Kimmel on his TV show. The study reports a surge in interest in the disease following the airing of the show and concludes that TV talk shows are an effective way to raise awareness of rare diseases.

The manuscript is well written and clear, the results and conclusion are supported by the data presented.

\section{Score Card}

Presentation

5.0

Is the article written in clear and proper English? (30\%)

Is the data presented in the most useful manner? (40\%)

Does the paper cite relevant and related articles appropriately? (30\%)

Context

5.0

Does the title suitably represent the article? (25\%)

Does the abstract correctly embody the content of the article? (25\%)

Does the introduction give appropriate context? (25\%)

Is the objective of the experiment clearly defined? (25\%)

Analysis

5.0

Does the discussion adequately interpret the results presented? (40\%)

Is the conclusion consistent with the results and discussion? (40\%)

Are the limitations of the experiment as well as the contributions of the experiment clearly outlined? $(20 \%)$ 